PROCEEDINGS OF THE

AMERICAN MATHEMATICAL SOCIETY

Volume 127, Number 4, April 1999, Pages 1243-1249

S 0002-9939(99)04685-7

\title{
REMARK ABOUT HEAT DIFFUSION ON PERIODIC SPACES
}

\author{
JOHN LOTT
}

(Communicated by Jozef Dodziuk)

\begin{abstract}
Let $M$ be a complete Riemannian manifold with a free cocompact $\mathbb{Z}^{k}$-action. Let $k\left(t, m_{1}, m_{2}\right)$ be the heat kernel on $M$. We compute the asymptotics of $k\left(t, m_{1}, m_{2}\right)$ in the limit in which $t \rightarrow \infty$ and $d\left(m_{1}, m_{2}\right) \sim \sqrt{t}$. We show that in this limit, the heat diffusion is governed by an effective Euclidean metric on $\mathbb{R}^{k}$ coming from the Hodge inner product on $\mathrm{H}^{1}\left(M / \mathbb{Z}^{k} ; \mathbb{R}\right)$.
\end{abstract}

\section{INTRODUCTION}

Let $M$ be a complete connected oriented $n$-dimensional Riemannian manifold. Let $k\left(t, m_{1}, m_{2}\right)$ be the time- $t$ heat kernel on $M$. The usual ansatz to approximate $k\left(t, m_{1}, m_{2}\right)$ is to say that

$$
k\left(t, m_{1}, m_{2}\right) \sim P\left(t, m_{1}, m_{2}\right) e^{-\frac{d\left(m_{1}, m_{2}\right)^{2}}{4 t}}
$$

where $e^{-\frac{d\left(m_{1}, m_{2}\right)^{2}}{4 t}}$ is considered to be the leading term and $P\left(t, m_{1}, m_{2}\right)$ is a correction term which can be computed iteratively. There are results which make this precise. For example [1], if $m_{1}$ and $m_{2}$ are nonconjugate, then as $t \rightarrow 0$,

$$
k\left(t, m_{1}, m_{2}\right)=\sum_{\gamma} \frac{\left(\operatorname{det} d\left(\exp _{m_{1}}\right)_{v_{\gamma}}\right)^{-1 / 2}}{(4 \pi t)^{n / 2}} e^{-\frac{d\left(m_{1}, m_{2}\right)^{2}}{4 t}}(1+O(t)) .
$$

Here the sum is over minimal geodesics $\gamma:[0,1] \rightarrow M$ joining $m_{1}$ to $m_{2}$ of the form $\gamma(s)=\exp _{m_{1}}\left(s v_{\gamma}\right)$. For another example, if $M$ has bounded geometry, then lower and upper heat kernel bounds [4], [5] imply that (1.1) is a good approximation if $d\left(m_{1}, m_{2}\right)>>t$, in the sense that $-\ln \left(k\left(t, m_{1}, m_{2}\right)\right)$ is well-approximated by $\frac{d\left(m_{1}, m_{2}\right)^{2}}{4 t}$.

One can ask if the ansatz (1.1) is relevant for other asymptotic regimes. In this paper we look at the case when $M$ has a periodic metric, meaning that $\mathbb{Z}^{k}$ acts freely by orientation-preserving isometries on $M$, with $X=M / \mathbb{Z}^{k}$ compact. We consider the asymptotic regime in which $t \rightarrow \infty$ and $d\left(m_{1}, m_{2}\right) \sim \sqrt{t}$. As the typical time-t Brownian path will travel a distance comparable to $\sqrt{t}$, this is the regime which contains the bulk of the diffusing heat. We show that, in this regime, (1.1) is no longer a valid approximation. Instead, the heat diffusion is governed by

Received by the editors August 5, 1997.

1991 Mathematics Subject Classification. Primary 58G11.

Research supported by NSF grant DMS-9704633.

(C)1999 American Mathematical Society 
an effective Euclidean metric on $\mathbb{R}^{k}$. This metric is constructed using the Hodge inner product on $\mathrm{H}^{1}(X ; \mathbb{R})$.

To state the precise result, let $\mathcal{F}$ be a fundamental domain in $M$ for the $\mathbb{Z}^{k}$ action. Given $\mathbf{v} \in \mathbb{Z}^{k}$, put

$$
k(t, \mathbf{v})=\int_{\mathcal{F}} k(t, m, \mathbf{v} \cdot m) d \operatorname{vol}(m) .
$$

This is independent of the choice of fundamental domain $\mathcal{F}$.

The covering $M \rightarrow X$ is classified by a map $\nu: X \rightarrow B \mathbb{Z}^{k}$, defined up to homotopy, which is $\pi_{1}$-surjective. It induces a surjection $\nu_{*}: \mathrm{H}_{1}(X ; \mathbb{R}) \rightarrow \mathbb{R}^{k}$ and an injection $\nu^{*}:\left(\mathbb{R}^{k}\right)^{*} \rightarrow \mathrm{H}^{1}(X ; \mathbb{R})$. Let $\langle\cdot, \cdot\rangle_{H^{1}(X ; \mathbb{R})}$ be the Hodge inner product on $\mathrm{H}^{1}(X ; \mathbb{R})$.

Definition 1. The inner product $\langle\cdot, \cdot\rangle_{\left(\mathbb{R}^{k}\right)^{*}}$ on $\left(\mathbb{R}^{k}\right)^{*}$ is given by

$$
\langle\cdot, \cdot\rangle_{\left(\mathbb{R}^{k}\right)^{*}}=\frac{\left(\nu^{*}\right)^{*}\langle\cdot, \cdot\rangle_{H^{1}(X ; \mathbb{R})}}{\operatorname{vol}(X)} .
$$

The inner product $\langle\cdot, \cdot\rangle_{\mathbb{R}^{k}}$ is the dual inner product on $\mathbb{R}^{k}$.

Let $\operatorname{vol}\left(\mathbb{R}^{k} / \mathbb{Z}^{k}\right)$ be the volume of a lattice cell in $\mathbb{R}^{k}$, measured with $\langle\cdot, \cdot\rangle_{\mathbb{R}^{k}}$.

Proposition 1. Fix $C>0$. Then in the region $\left\{(t, \mathbf{v}) \in \mathbb{R}^{+} \times \mathbb{Z}^{k}:\langle\mathbf{v}, \mathbf{v}\rangle_{\mathbb{R}^{k}} \leq C t\right\}$, as $t \rightarrow \infty$ we have

$$
k(t, \mathbf{v})=\frac{\operatorname{vol}\left(\mathbb{R}^{k} / \mathbb{Z}^{k}\right)}{(4 \pi t)^{k / 2}} e^{-\langle\mathbf{v}, \mathbf{v}\rangle_{\mathbb{R}^{k}} /(4 t)}+O\left(t^{-\frac{k+1}{2}}\right)
$$

uniformly in $\mathbf{v}$.

Example. 1. If $M=\mathbb{R}^{k}$ with a flat metric $\langle\cdot, \cdot\rangle_{\text {flat }}$, then one can check that $\langle\cdot, \cdot\rangle_{\mathbb{R}^{k}}=\langle\cdot, \cdot\rangle_{\text {flat }}$, so one recovers the standard flat-space heat kernel.

2. If $n=2$, then $\langle\cdot, \cdot\rangle_{H^{1}(X ; \mathbb{R})}$ is conformally-invariant. Hence in this case, the heat kernel asymptotics only depend on $\operatorname{vol}(X)$ and the induced complex structure on $X$.

One can get similar pointwise estimates on $k\left(t, m_{1}, m_{2}\right)$ by the same methods. We omit the details.

The result of Proposition 1 is an example of the phenomenon of "homogenization", which has been much-studied for differential operators on $\mathbb{R}^{n}$. Homogenization means that in an appropriate scaling limit, the solution to a problem is governed by the solution to a spatially homogeneous problem; see [2] and references therein. Thus it is not surprising that the answer in Proposition 1 has a homogeneous form. The point of the present paper is to show how one can compute the exact asymptotics in the general geometric setting.

We remark that when $t \rightarrow \infty$ and $d\left(m_{1}, m_{2}\right)>>t$, the asymptotic expression (1.1) also shows homogenization. This follows from the result of D. Burago [3] that there are a Banach norm $\|\cdot\|$ on $\mathbb{R}^{k}$ and a constant $c>0$ such that if $m \in M$ and $\mathbf{v} \in \mathbb{Z}^{k}$, then $|d(m, \mathbf{v} \cdot m)-\|\mathbf{v}\|| \leq c$. Thus as $t \rightarrow \infty$, if $d\left(m_{1}, m_{2}\right) \sim \sqrt{t}$, then the effective geometry is $\left(\mathbb{R}^{k},\langle\cdot, \cdot\rangle_{\mathbb{R}^{k}}\right)$, while if $d\left(m_{1}, m_{2}\right)>>t$, then the effective geometry is $\left(\mathbb{R}^{k},\|\cdot\|\right)$.

It would be interesting if one could extend the results of this paper to the setting in which $\Gamma$ is a nonabelian discrete group, such as the fundamental group of a closed hyperbolic surface. In this case, the relevant scaling regime should be $t \rightarrow \infty$ and 
$d\left(m_{1}, m_{2}\right) \sim t$, as the typical time- $t$ Brownian path on the hyperbolic plane travels a distance comparable to $t$.

I thank the IHES for its hospitality while this work was done and Palle Jorgensen for sending his reprints.

\section{Proof of Proposition 1}

We first recall some basic facts about the eigenvalues of a parametrized family of operators [7, Chapter XII].

Let $M_{d}(\mathbb{C})$ be the vector space of $d \times d$ complex matrices and let $M_{d}^{s a}(\mathbb{C})$ be the subspace of self-adjoint matrices. Let $f: \mathbb{R}^{k} \rightarrow M_{d}(\mathbb{C})$ be a real-analytic map. The eigenvalues $\left\{\lambda_{i}(x)\right\}_{i=1}^{d}$ of $f(x)$ are algebraic functions of $x$, meaning the roots of a polynomial whose coefficients are real-analytic functions of $x$, as they are given by $\operatorname{det}(f(x)-\lambda)=0$. If $\lambda_{1}(0)$ is a nondegenerate eigenvalue of $f(0)$, then it extends near $x=0$ to a real-analytic function $\lambda_{1}(x)$.

If $k=1$ and $f$ takes values in $M_{d}^{s a}(\mathbb{C})$, then the eigenvalues of $f$ form $d$ realanalytic functions $\left\{\lambda_{i}(x)\right\}_{i=1}^{d}$ on $\mathbb{R}$. Of course, these functions may cross. If $k>$ 1 and $f$ takes values in $M_{d}^{s a}(\mathbb{C})$, then it may not be true that the eigenvalues form real-analytic functions on $\mathbb{R}^{k}$. This can be seen in the example $f\left(x_{1}, x_{2}\right)=$ $\left(\begin{array}{cc}0 & x_{1}-i x_{2} \\ x_{1}+i x_{2} & 0\end{array}\right)$. Its eigenvalues are $\pm \sqrt{x_{1}^{2}+x_{2}^{2}}$, which are not the union of two smooth functions on $\mathbb{R}^{2}$. However, if $\gamma(s)$ is a real-analytic curve in $\mathbb{R}^{2}$, then the eigenvalues of $f(\gamma(s))$ do form real-analytic functions in $s$.

If $f$ is instead an appropriate real-analytic family of operators on a Hilbert space, then one has similar results. We refer to [7, Chapter XII.2] for the precise requirements.

To prove Proposition 1, we use the method of [6, Section VI]. The Pontryagin dual of $\mathbb{Z}^{k}$ is $T^{k}=\left(\mathbb{R}^{k}\right)^{*} / 2 \pi\left(\mathbb{Z}^{k}\right)^{*}$. Given $\theta \in T^{k}$, let $\rho(\theta): \mathbb{Z}^{k} \rightarrow U(1)$ be the corresponding representation and let $E(\theta)$ be the flat line bundle on $X$ associated to the representation $\pi_{1}(X) \stackrel{\nu_{*}}{\rightarrow} \mathbb{Z}^{k} \stackrel{\rho(\theta)}{\rightarrow} U(1)$. Let $\triangle_{\theta}$ be the Laplacian on $L^{2}(X ; E(\theta))$. Then Fourier analysis gives

$$
k(t, \mathbf{v})=\int_{T^{k}} e^{i \theta \cdot \mathbf{v}} \operatorname{Tr}\left(e^{-t \triangle(\theta)}\right) \frac{d^{k} \theta}{(2 \pi)^{k}}
$$

Now $\operatorname{Ker}(\triangle(\theta))=0$ if $\theta \neq 0$ and $\operatorname{Ker}(\triangle(0)) \cong \mathbb{C}$ consists of the constant functions on $X$.

In order to write all of the operators $\triangle(\theta)$ as acting on the same Hilbert space, let $\left\{\tau^{j}\right\}_{j=1}^{k}$ be a set of harmonic 1-forms on $X$ which gives an integral basis of $\left(\mathbb{Z}^{k}\right)^{*} \subset\left(\mathbb{R}^{k}\right)^{*} \subseteq \mathrm{H}^{1}(X ; \mathbb{R})$. Let $e\left(\tau^{j}\right)$ denote exterior multiplication by $\tau^{j}$ on $C^{\infty}(X)$ and let $i\left(\tau^{j}\right)$ denote interior multiplication by $\tau^{j}$ on $\Omega^{1}(X)$. Putting

$$
d(\theta)=d+i \sum_{j=1}^{k} \theta_{j} e\left(\tau^{j}\right)
$$

and

$$
d^{*}(\theta)=d^{*}-i \sum_{j=1}^{k} \theta_{j} i\left(\tau^{j}\right),
$$

$\triangle(\theta)$ is unitarily equivalent to the self-adjoint operator $d^{*}(\theta) d(\theta)$ (which we shall also denote by $\triangle(\theta))$ acting on $L^{2}(X)$. Because $\triangle(\theta)$ is quadratic in $\theta$, it is easy 
to see that $\{\triangle(\theta)\}_{\theta \in T^{k}}$ is an analytic family of type (A) in the sense of [7, Chapter XII.2], so we can apply analytic eigenvalue perturbation theory. In particular, if $\left\{\lambda_{i}(\theta)\right\}_{i \in \mathbb{Z}^{+}}$are the eigenvalues of $\triangle(\theta)$, arranged in increasing order and repeated if there is a multipicity greater than one, then $\lambda_{1}(\theta) \geq 0$ and $\lambda_{1}(\theta)=0$ if and only if $\theta=0$, in which case it is a nondegenerate eigenvalue. Thus $\lambda_{1}$ extends to a real-analytic function in a neighborhood of $\theta=0$. So for sufficiently small $\epsilon>0$, there is a neighborhood $U \subseteq T^{k}$ of $0 \in T^{k}$ such that

1. If $\theta \notin U$, then $\lambda_{1}(\theta)>\epsilon$.

2. Restricted to $U, \lambda_{1}$ is a real-analytic function which represents a nondegenerate eigenvalue and $\lambda_{2}>\epsilon$.

From (2.1), we have

$$
k(t, \mathbf{v})=\int_{T^{k}} e^{i \theta \cdot \mathbf{v}} \sum_{i=1}^{\infty} e^{-t \lambda_{i}(\theta)} \frac{d^{k} \theta}{(2 \pi)^{k}} .
$$

Then it is easy to show that

$$
k(t, \mathbf{v})=\int_{U} e^{i \theta \cdot \mathbf{v}} e^{-t \lambda_{1}(\theta)} \frac{d^{k} \theta}{(2 \pi)^{k}}+O\left(e^{-\epsilon t / 2}\right)
$$

uniformly in $\mathbf{v}$.

Lemma 1. The Taylor's series of $\lambda_{1}(\theta)$ near $\theta=0$ starts off as

$$
\lambda_{1}(\theta)=\langle\theta, \theta\rangle_{\left(\mathbb{R}^{k}\right)^{*}}+O\left(|\theta|^{3}\right) .
$$

Proof. It suffices to compute $\left.\frac{d \lambda_{1}(s \vec{w})}{d s}\right|_{s=0}$ and $\left.\frac{d^{2} \lambda_{1}(s \vec{w})}{d s^{2}}\right|_{s=0}$ for all $\vec{w} \in\left(\mathbb{R}^{k}\right)^{*}$. For simplicity, denote $\triangle(s \vec{w})$ by $\triangle(s)$ and $\lambda_{1}(s \vec{w})$ by $\lambda(s)$. As $\lambda(s)$ is nonnegative and $\lambda(0)=0$, we must have $\lambda^{\prime}(0)=0$. Let $\psi(s)$ denote a nonzero eigenfunction with eigenvalue $\lambda(s)$; we can assume that it is real-analytic in $s$ with $\psi(0)=1$. Differentiation of $\triangle(s) \psi(s)=\lambda(s) \psi(s)$ gives

$$
\triangle^{\prime}(0) \psi(0)+\triangle(0) \psi^{\prime}(0)=0
$$

and

$$
\triangle^{\prime \prime}(0) \psi(0)+2 \triangle^{\prime}(0) \psi^{\prime}(0)+\triangle(0) \psi^{\prime \prime}(0)=\lambda^{\prime \prime}(0) \psi(0)
$$

Taking the inner product of $(2.8)$ with $\psi(0)$ gives

$$
\left\langle\psi(0), \triangle^{\prime \prime}(0) \psi(0)\right\rangle+2\left\langle\psi(0), \triangle^{\prime}(0) \psi^{\prime}(0)\right\rangle=\lambda^{\prime \prime}(0)\langle\psi(0), \psi(0)\rangle .
$$

Let $G$ be the Green's operator for $\triangle(0)$. From (2.7),

$$
\psi^{\prime}(0)=c \psi(0)-G \triangle^{\prime}(0) \psi(0)
$$

for some constant $c$. Changing $\psi(s)$ to $e^{-c s} \psi(s)$, we may assume that $c=0$. Substituting (2.10) into (2.9) gives

$$
\left\langle\psi(0), \triangle^{\prime \prime}(0) \psi(0)\right\rangle-2\left\langle\psi(0), \triangle^{\prime}(0) G \triangle^{\prime}(0) \psi(0)\right\rangle=\lambda^{\prime \prime}(0)\langle\psi(0), \psi(0)\rangle .
$$

It remains to compute $\left\langle\psi(0), \triangle^{\prime \prime}(0) \psi(0)\right\rangle$ and $\left\langle\psi(0), \triangle^{\prime}(0) G \triangle^{\prime}(0) \psi(0)\right\rangle$. Put $D(s)=d_{s \vec{w}}$ and $D^{*}(s)=d_{s \vec{w}}^{*}$. Then $\triangle(s)=D^{*}(s) D(s)$. From (2.2) and (2.3), $D(s)$ and $D^{*}(s)$ are linear in $s$, with

$$
D^{\prime}(0)=i \sum_{j=1}^{k} w_{j} e\left(\tau^{j}\right)
$$


and

$$
\left(D^{*}\right)^{\prime}(0)=-i \sum_{j=1}^{k} w_{j} i\left(\tau^{j}\right)
$$

Then

$$
\begin{aligned}
\left\langle\psi(0), \triangle^{\prime \prime}(0) \psi(0)\right\rangle & =2\left\langle\psi(0),\left(D^{*}\right)^{\prime}(0) D^{\prime}(0) \psi(0)\right\rangle \\
& =2\left|D^{\prime}(0) \psi(0)\right|_{H^{1}(X ; \mathbb{C})}^{2} \\
& =2\left|\sum_{j=1}^{k} w_{j} \tau^{j}\right|_{H^{1}(X ; \mathbb{C})}^{2}
\end{aligned}
$$

Now

$$
\begin{aligned}
\triangle^{\prime}(0) \psi(0) & =\left[\left(D^{*}\right)^{\prime}(0) D(0)+D^{*}(0) D^{\prime}(0)\right] \psi(0) \\
& =d^{*}\left(-i \sum_{j=1}^{k} w_{j} \tau^{j}\right)=0 .
\end{aligned}
$$

Substituting (2.14) and (2.15) into (2.11) and using the fact that $\langle\psi(0), \psi(0)\rangle=$ $\operatorname{vol}(X)$, the lemma follows.

Continuing with the proof of Proposition 1, by Morse theory and Lemma 1, we can find a change of coordinates near $0 \in T^{k}$ with respect to which $\lambda_{1}$ becomes quadratic. That is, if $B_{r}(0)$ denotes the ball of radius $r$ in $\left(\mathbb{R}^{k}\right)^{*}$, we can find an $r>0$, a neighborhood $U$ of $0 \in T^{k}$ and a diffeomorphism $\phi: B_{r}(0) \rightarrow U$ such that $\phi(0)=0, d \phi_{0}=\mathrm{Id}$ and $\lambda_{1}(\phi(x))=\langle x, x\rangle_{\left(\mathbb{R}^{k}\right)^{*}}$. Then there is some $\alpha>0$ such that as $t \rightarrow \infty$,

$$
k(t, \mathbf{v})=\int_{B_{r}(0)} e^{i \phi(x) \cdot \mathbf{v}} e^{-t\langle x, x\rangle_{\left(\mathbb{R}^{k}\right)^{*}}} \operatorname{det}\left(d \phi_{x}\right) \frac{d^{k} x}{(2 \pi)^{k}}+O\left(e^{-\alpha t}\right),
$$

uniformly in $\mathbf{v}$. Multiplying by a cutoff function on $\left(\mathbb{R}^{k}\right)^{*}$, we can write

$$
\begin{aligned}
k(t, \mathbf{v}) & =\int_{\left(\mathbb{R}^{k}\right)^{*}} e^{i \phi(x) \cdot \mathbf{v}} e^{-t\langle x, x\rangle_{\left(\mathbb{R}^{k}\right)^{*}}} g(x) \frac{d^{k} x}{(2 \pi)^{k}}+O\left(e^{-\alpha^{\prime} t}\right) \\
& =t^{-\frac{k}{2}} \int_{\left(\mathbb{R}^{k}\right)^{*}} e^{i \phi\left(\frac{x}{\sqrt{t}}\right) \cdot \mathbf{v}} e^{-\langle x, x\rangle_{(\mathbb{R})^{*}}} g\left(\frac{x}{\sqrt{t}}\right) \frac{d^{k} x}{(2 \pi)^{k}}+O\left(e^{-\alpha^{\prime} t}\right)
\end{aligned}
$$

for some $g \in C_{0}^{\infty}\left(\left(\mathbb{R}^{k}\right)^{*}\right)$ with $g(0)=1$ and some $\alpha^{\prime}>0$. (Here $\phi$ has been extended to become a map $\phi:\left(\mathbb{R}^{k}\right)^{*} \rightarrow\left(\mathbb{R}^{k}\right)^{*}$ which is the identity outside of a compact set.)

We have now reduced to a stationary-phase-type integral. Let

$$
g(x)=1+(\nabla g)(0) \cdot x+E(x)
$$


be the beginning of the Taylor's expansion of $g$. We can write

$$
\begin{aligned}
t^{-\frac{k}{2}} & \int_{\left(\mathbb{R}^{k}\right)^{*}} e^{i \phi\left(\frac{x}{\sqrt{t}}\right) \cdot \mathbf{v}} e^{-\langle x, x\rangle_{(\mathbb{R})^{*}}} g\left(\frac{x}{\sqrt{t}}\right) \frac{d^{k} x}{(2 \pi)^{k}} \\
= & t^{-\frac{k}{2}} \int_{\left(\mathbb{R}^{k}\right)^{*}} e^{i \frac{x}{\sqrt{t}} \cdot \mathbf{v}} e^{-\langle x, x\rangle_{\left(\mathbb{R}^{k}\right)^{*}}}\left[1+(\nabla g)(0) \cdot \frac{x}{\sqrt{t}}+E\left(\frac{x}{\sqrt{t}}\right)\right] \frac{d^{k} x}{(2 \pi)^{k}} \\
& +t^{-\frac{k}{2}} \int_{\left(\mathbb{R}^{k}\right)^{*}} e^{i \frac{x}{\sqrt{t}} \cdot \mathbf{v}}\left[e^{i\left(\phi\left(\frac{x}{\sqrt{t}}\right)-\frac{x}{\sqrt{t}}\right) \cdot \mathbf{v}}-1\right] e^{-\langle x, x\rangle_{\left(\mathbb{R}^{k}\right)^{*}}} g\left(\frac{x}{\sqrt{t}}\right) \frac{d^{k} x}{(2 \pi)^{k}}
\end{aligned}
$$

Recall that the measure $\frac{d^{k} x}{(2 \pi)^{k}}$ on $\left(\mathbb{R}^{k}\right)^{*}$ derives from the product measure on $T^{k}=\left(\mathbb{R}^{*} / 2 \pi \mathbb{Z}^{*}\right)^{k}$. Let $\langle\cdot, \cdot\rangle_{\text {prod }}$ be the standard product Euclidean metric on $\left(\mathbb{R}^{*}\right)^{k}$. Let $Q$ be the self-adjoint operator on $\left(\mathbb{R}^{k}\right)^{*}$ such that $\langle x, x\rangle_{\left(\mathbb{R}^{k}\right)^{*}}=\langle x, Q x\rangle_{\text {prod }}$. Then a standard calculation gives

$$
t^{-\frac{k}{2}} \int_{\left(\mathbb{R}^{k}\right)^{*}} e^{i \frac{x}{\sqrt{t}} \cdot \mathbf{v}} e^{-\langle x, x\rangle_{\left(\mathbb{R}^{k}\right)^{*}}} \frac{d^{k} x}{(2 \pi)^{k}}=\frac{(\operatorname{det} Q)^{-1 / 2}}{(4 \pi t)^{k / 2}} e^{-\langle\mathbf{v}, \mathbf{v}\rangle_{\mathbb{R}^{k}} /(4 t)} .
$$

On the other hand,

$$
(\operatorname{det} Q)^{-1 / 2}=\operatorname{vol}\left(\mathbb{R}^{k} / \mathbb{Z}^{k}\right)
$$

By symmetry,

$$
t^{-\frac{k}{2}} \int_{\left(\mathbb{R}^{k}\right)^{*}} e^{i \frac{x}{\sqrt{t}} \cdot \mathbf{v}} e^{-\langle x, x\rangle_{\left(\mathbb{R}^{k}\right)^{*}}}(\nabla g)(0) \cdot \frac{x}{\sqrt{t}} \frac{d^{k} x}{(2 \pi)^{k}}=0 .
$$

Let $c>0$ be such that $|E(x)| \leq c\langle x, x\rangle_{\left(\mathbb{R}^{k}\right)^{*}}$ for all $x \in\left(\mathbb{R}^{k}\right)^{*}$. Then

$$
\begin{aligned}
& \left|\int_{\left(\mathbb{R}^{k}\right)^{*}} e^{i \frac{x}{\sqrt{t}} \cdot \mathbf{v}} e^{-\langle x, x\rangle_{(\mathbb{R})^{*}}} E\left(\frac{x}{\sqrt{t}}\right) \frac{d^{k} x}{(2 \pi)^{k}}\right| \\
& \quad \leq \frac{c}{t} \int_{\left(\mathbb{R}^{k}\right)^{*}}\langle x, x\rangle_{\left(\mathbb{R}^{k}\right)^{*}} e^{-\langle x, x\rangle_{(\mathbb{R})^{k}}} \frac{d^{k} x}{(2 \pi)^{k}} .
\end{aligned}
$$

Finally,

$$
\begin{aligned}
& \left|\int_{\left(\mathbb{R}^{k}\right)^{*}} e^{i \frac{x}{\sqrt{t}} \cdot \mathbf{v}}\left[e^{i\left(\phi\left(\frac{x}{\sqrt{t}}\right)-\frac{x}{\sqrt{t}}\right) \cdot \mathbf{v}}-1\right] e^{-\langle x, x\rangle_{(\mathbb{R} k)^{*}}} g\left(\frac{x}{\sqrt{t}}\right) \frac{d^{k} x}{(2 \pi)^{k}}\right| \\
& \quad \leq\|g\|_{\infty} \int_{\left(\mathbb{R}^{k}\right)^{*}} 2\left|\sin \left(\frac{1}{2}\left[\phi\left(\frac{x}{\sqrt{t}}\right)-\frac{x}{\sqrt{t}}\right] \cdot \mathbf{v}\right)\right| e^{-\langle x, x\rangle_{(\mathbb{R})^{*}}} \frac{d^{k} x}{(2 \pi)^{k}} .
\end{aligned}
$$

We can find a constant $c^{\prime}>0$ such that

$$
2\left|\sin \left(\frac{1}{2}[\phi(x)-x] \cdot \mathbf{v}\right)\right| \leq c^{\prime}\langle x, x\rangle_{\left(\mathbb{R}^{k}\right)^{*}}\|\mathbf{v}\|_{\mathbb{R}^{k}}
$$


for all $x \in\left(\mathbb{R}^{k}\right)^{*}$ and $\mathbf{v} \in \mathbb{Z}^{k}$. Then

$$
\begin{gathered}
\|g\|_{\infty} \int_{\left(\mathbb{R}^{k}\right)^{*}} 2\left|\sin \left(\frac{1}{2}\left[\phi\left(\frac{x}{\sqrt{t}}\right)-\frac{x}{\sqrt{t}}\right] \cdot \mathbf{v}\right)\right| e^{-\langle x, x\rangle_{\left(\mathbb{R}^{k}\right)^{*}}} \frac{d^{k} x}{(2 \pi)^{k}} \\
\leq \frac{c^{\prime}}{\sqrt{t}} \frac{\|\mathbf{v}\|_{\mathbb{R}^{k}}}{\sqrt{t}}\|g\|_{\infty} \int_{\left(\mathbb{R}^{k}\right)^{*}}\langle x, x\rangle_{\left(\mathbb{R}^{k}\right)^{*}} e^{-\langle x, x\rangle_{\left(\mathbb{R}^{k}\right)^{*}}} \frac{d^{k} x}{(2 \pi)^{k}} .
\end{gathered}
$$

By assumption,

$$
\frac{\|\mathbf{v}\|_{\mathbb{R}^{k}}}{\sqrt{t}} \leq \sqrt{C} .
$$

The proposition follows from combining equations (2.17)-(2.27).

\section{REFERENCES}

[1] R. Azencott et al., Géodesiques et Diffusions en Temps Petit, Astérisque 84-85, Société Mathématique de France, Paris (1981)

[2] C. Batty, O. Bratteli, P. Jorgensen and D. Robinson, "Asymptotics of Periodic Subelliptic Operators", J. of Geom. Anal. 5, p. 427-443 (1995) MR 97f:35028

[3] D. Burago, "Periodic Metrics", in Advances in Soviet Math. 9, p. 241-248 (1992) MR 93c:53029

[4] J. Cheeger and S.-T. Yau, "A Lower Bound for the Heat Kernel", Comm. Pure Appl. Math. 34, p. 465-480 (1981) MR 82i:58065

[5] E. Davies and M. Pang, "Sharp Heat Kernel Bounds for some Laplace Operators", Quart. J. Math. Oxford 40, p. 281-290 (1989) MR 91i:58142

[6] J. Lott, "Heat Kernels on Covering Spaces and Topological Invariants", J. Diff. Geom. 35, p. 471-510 (1992) MR 93b:58140

[7] M. Reed and B. Simon, Methods of Mathematical Physics, Academic Press, New York (1978)

Department of Mathematics, University of Michigan, Ann Arbor, Michigan 481091109

E-mail address: lott@math.1sa.umich.edu 